\title{
vibrations générées dans le sol par une installation de concassage
}

\author{
par \\ F. H. Cornet \\ Institut de Physique du Globe, Paris \\ P. Habib \\ École Polytechnique, Palaiseau
}

RESUME - Les résultats d'une campagne de mesure des vibrations générëes dans le sol par l'exploitation d'une carrière à ciel ouvert sont présentés. Deux types de mouvements ont été étudiés, à savoir les mouvements stationnaires liés â l'activité des unités de concassage et les mouvements transitoires liés aux tirs de mine. Les premiers sont ressentis au moins jusqu'à $3,5 \mathrm{~km}$ de la carrière, mais ne sont plus discernables du bruit de fond à $7 \mathrm{~km}$. Les seconds $n^{\prime}$ ont été étudiés que pour des distances du point de tir supérieures ou égales à $1 \mathrm{~km}$; dans ces domaines de distance, les terrains conservent un comportement élastique pour des tirs de $535 \mathrm{~kg}$ de dynamite avec 6 micro-retards de 0,005 seconde.

\section{INTRODUCTION}

Cette étude a pour but de décrire les mouvements vibratoires générés dans le sol du fait de l'exploitation d'une carrière à ciel ouvert.

Deux types de mouvements ont étê étudiés : - les mouvements stationnaires Tiês à T'activitẽ des unitês de concassage (6000 t/jour).

- les mouvements transitoires liés aux tirs de mines.

Plus précisément, il s'agissait de déterminer si les mouvements stationnaires étaient inférieurs à 0,01 micron à $7 \mathrm{~km}$ de $1 \mathrm{a}$ carrière, amplitude maximum tolérée pour le bon fonctionnement d'un appareillage d'observation interférométrique d'astronomie.

La carrière est implantée sur la commune de Roquefort les Pins (Alpes-maritimes) sur des calcaires marmoréens du Berriasien-Portlandien. L'étude de la propagation des ondes a été effectuée dans un secteur d'angle ếgal à $90^{\circ}$ au nord ouest de la carrière, sur des terrains essentiellement dolomitiques (kimméridgien et jurassique supérieur) et calcaires (Bajocien-Bathonien) avec toutefois des calcaires et marnes vertes du Rhétien pour les stations d'observation situées à $7 \mathrm{~km}$ à 1 'ouest de 1 a carrière (voir figure 1).

\section{ETUDE DES MOUVEMENTS STATIONNAIRES}

La limite inférieure de la bande passante pour laquelle l'étude a été effectuée a été choisie de façon à coîncider avec des demi-longueurs d'ondes égales à cing fois la plus grande longueur de base entre les capteurs utilisés pour les mesures interférométriques, c'est-à-dire à des lonqueurs d'onde de $2000 \mathrm{~m}$. L'enregistrement d'un tir de mine a permis de déterminer la vitesse de propagation des ondes $S\left(C_{c}\right)$ en surface ( $c^{\prime}$ est-à-dire la vitesse la plus proche de celle des ondes de Love susceptibles d'être générées par les concasseurs $\left(C_{L} \quad 0,9 C_{S}\right)$ ) soit $2500 \mathrm{~m} / \mathrm{s}$. La borne inférieure de la bande passante utile pour la présente étude a donc été choisie égale à $1 \mathrm{~Hz}$; 1a borne supérieure a été placée à $50 \mathrm{~Hz}$, valeur qui correspond à la limite imposée par la chaîne de numérisation utilisée pour analyser les enregistrements. Cette valeur s'est révêlée a posteriori, tout a fait satisfaisante.

\section{Description des mesures}

Le matériel de mesure mis en place sur le terrain était constitué d'un sismonètre trois composantes orienté de façon telle qu'un des géophones (réponse linéaire en vitesse dans le domaine 0,5-125 Hz) soit vertical ; que le deuxième soit dirigé dans la direction du système de concasseurs (composante longitudinale) et que le troisième soit perpendiculaire aux deux premiers (composante transversale) et d'une horloge émettant un signal toutes les secondes. Les signaux provenant des trois géophones, après avoîr été amplifiés, étaient modulês avec celui de l'horloge par rapport à une fréquence "porteuse" de 6,4 KHiz et le signal ainsi obtenu était enregistré sur magnétophone. A la démodulation cette fréquence porteuse permet d'asservir le moteur du magnétophone ce qui assure une restitution très précise de la vitesse de déroulement de la bande enregistrée.

Chaque station d'enregistrement a été occupée pendant 10 minutes ; cinq minutes correspondant à un gain de $2^{8}$ et les cinq autres minutes à un gain de $2^{9}$, ce qui assurait un enregistrement satisfaisant 

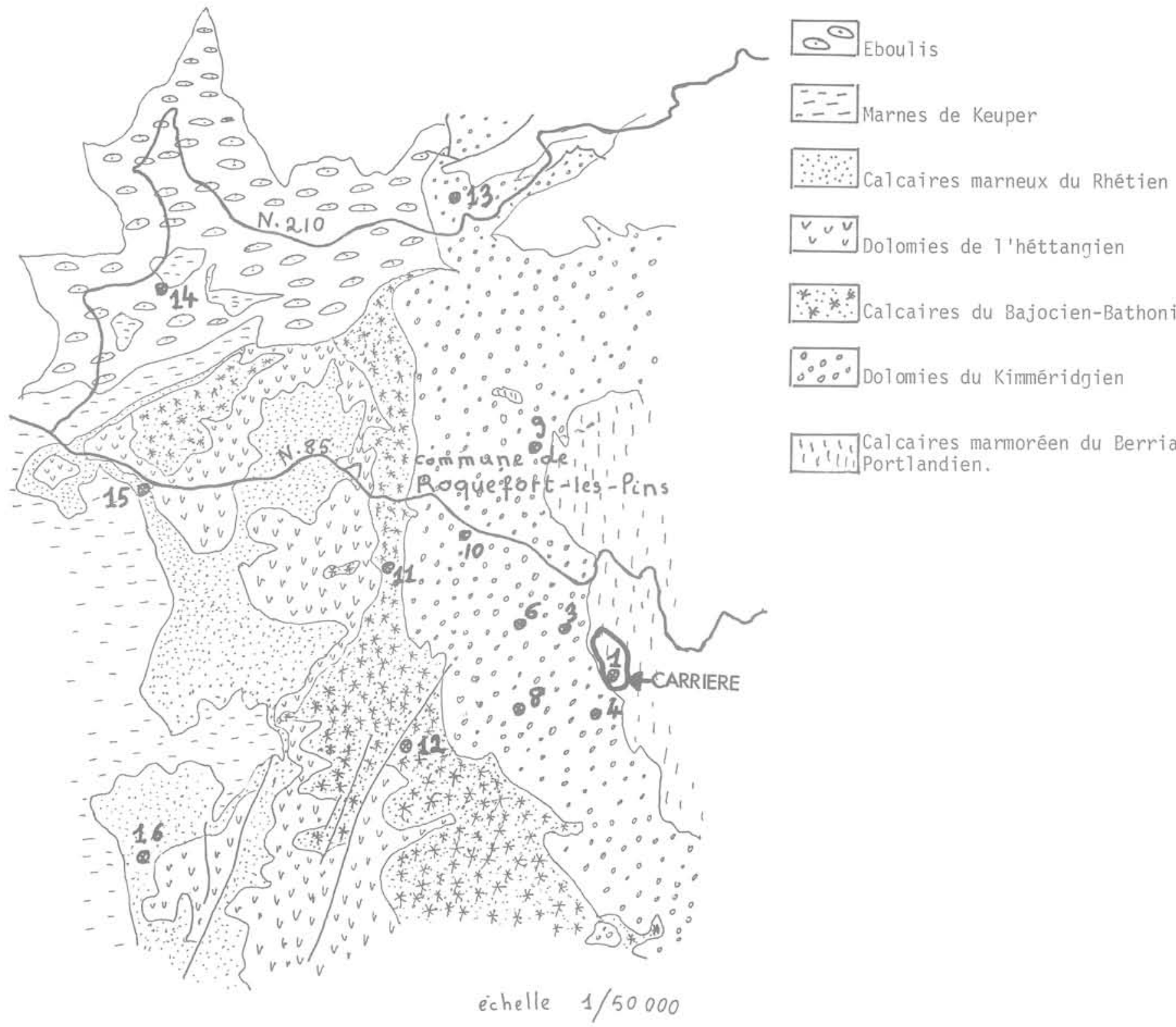

$v_{v} v v$ Dolomies de 1 'héttangien

$\because * * *$ Calcaires du Bajocien-Bathonien

$\because 00$ Dolomies du Kimméridgien 'ili, Calcaires marmorêen du Berriasien-
Portlandien.

Figure 1. - Disposition des stations d'enregistrement.

du bruit stationnaire. Il avait été initialement prévu de laisser de façon continue un sismomètre à $100 \mathrm{~m}$ du concasseur primaire et d'utiliser pour toutes les stations la même base de temps de façon à éviter d'étudier des "fenêtres" d'écoute pendant lesquelles le concasseur n'aurait pas fonctionné en régime normal. Malheureusement du fait de déficiences du magnétophone d'enregistrement de cette station de référence, seules 2 heures d'enregistrement continu ont pu être effectuées. Toutefois, nous avons pu vêrifier que les concasseurs ont fonctionné en régime normal durant la période d'étude grâce aux divers enregistrements effectués à moins de $3 \mathrm{~km}$ de la carriēre.

Les sites occupés pour les mesures ont été choisis de façon à obtenir des enregistrements à des distances de $500 \mathrm{~m}, 1 \mathrm{~km}, 3 \mathrm{~km}$ et $7 \mathrm{~km}$ pour quatre azimuths différents (voir figure 1). Les directions ont été choisies en fonction de la structure géologique de la région. En effet, la "pollution sismique" due aux concasseurs est probablement liēe essentiellement aux ondes de Love et ces ondes sont d'autant mieux développées que la vitesse de propagation des ondes dans le matériau superficiel est plus lente par rapport à celle des matériaux sous-jacents. (La vitesse des ondes dans un matêriau dépend des caractéristiques élastiques de celui-ci.).

\section{Analyse des enregistrements}

Dëfinition de la "signature sismique" de la carrière: A partir des dix minutes d'enregistrement continu obtenu pour chaque station, cinq "fenêtres" de huit secondes ont été sélectionnées pour leur aspect stationnaire : absence de mouvements transitoires liés au vent (très faible ce jour $1 a ̀$ ), au passage des voitures à proximité, ou toute autre source possible. Ces cinq fenêtres ont alors été numérisées et une analyse spectrale (par la méthode dite Fast Fourier Transform) dans le domaine 0,5-50 Hz, par pas de $0,9 \mathrm{~Hz}$, de chacune de ces fenêtres a été effectuée. 
Une étude comparêe des rêsultats obtenus pour les stations 1, 3 et 4 a permis de définir la "signature sismique" de la carrière. Pour ce faire, pour la station 1, supposant que les unités de concassage présentaient des fréquences propres assez bien marquées, nous avons repertorié les fréquences pour lesquelles un maximum relatif dans le spectre d'amplitude est observê. Les dix maxima les plus significatifs ont ainsi été cataloguês pour les cinq fenêtres d'échantillonage et pour les trois composantes. Il est alors apparu que ces maxima se trouvent systëmatiquement compris dans quatre domaines de fréquence relativement étroits : 8-10 Hz, $12 \mathrm{~Hz}, 15-18 \mathrm{~Hz}, 20-22 \mathrm{lz}$. De plus, une certaine quantitê d'énergie semble également gênêrẻe dans le domaine $33-38 \mathrm{~Hz}$ bien que les maxima d'amplitude que l'on y observe soient plus faibles que pour les quatre premières fenêtres (voir figure 2).

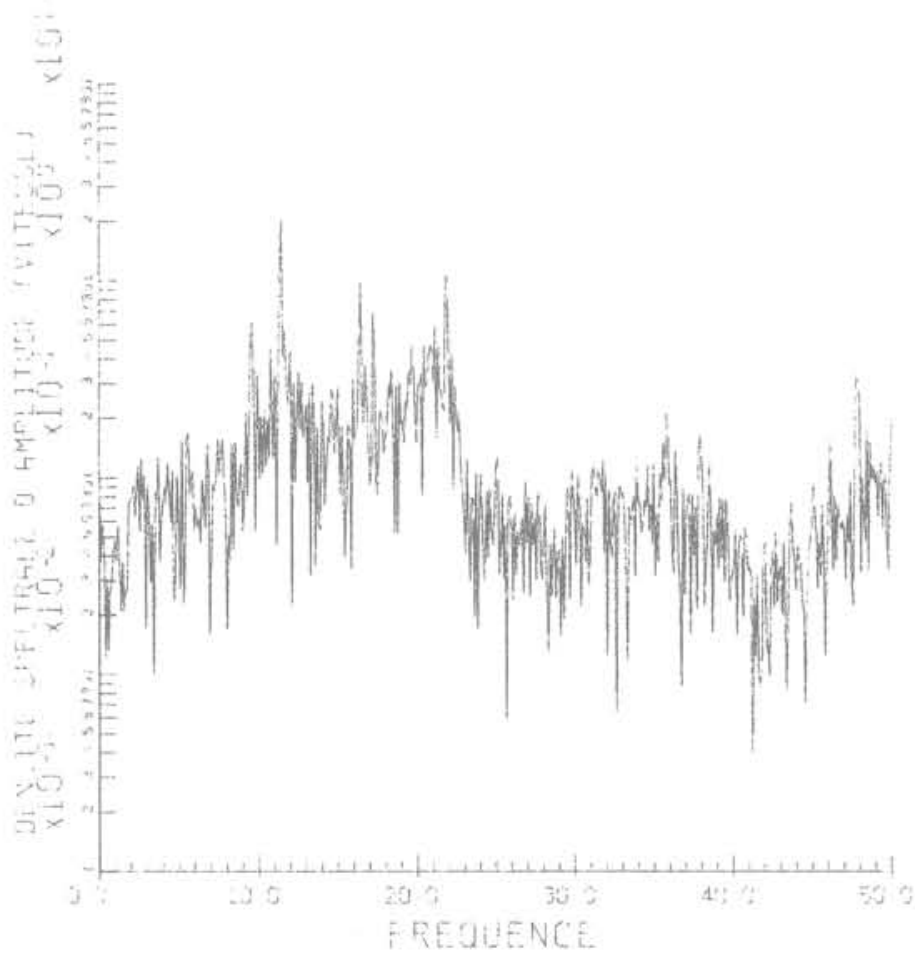

Figure 2. - Exemple de spectre d'amplitude pour la composante verticale des mouvement stationnaires de la station 1.

On notera de plus que les composantes horizontales présentent une amplitude plus importante pour ces fréquences que la composante verticale.

Il est à souligner que les mouvements vibratoires observês dans la carrière sont influencés non seulement par les unités de concassage mais aussi par le trafic de camions lourds qui y circulent. Nous avons donc conduit une étude similaire à la précédente sur la station 4 placẻe à $770 \mathrm{~m}$ de la carrière et par conséquent encore affectée de façon très significative par celle-ci, les bruit liês à 1a circulation des camions étant cependant nettement attênuês du fait du peu d'énergie qui leur est associée. Seule la composante verticale a été étudiée; on y retrouve très nettement les quatre domaines $8-10 \mathrm{~Hz}, 12 \mathrm{~Hz}, 16-18 \mathrm{~Hz}$ et $20-22 \mathrm{~Hz}$ (voir figure 3 ).

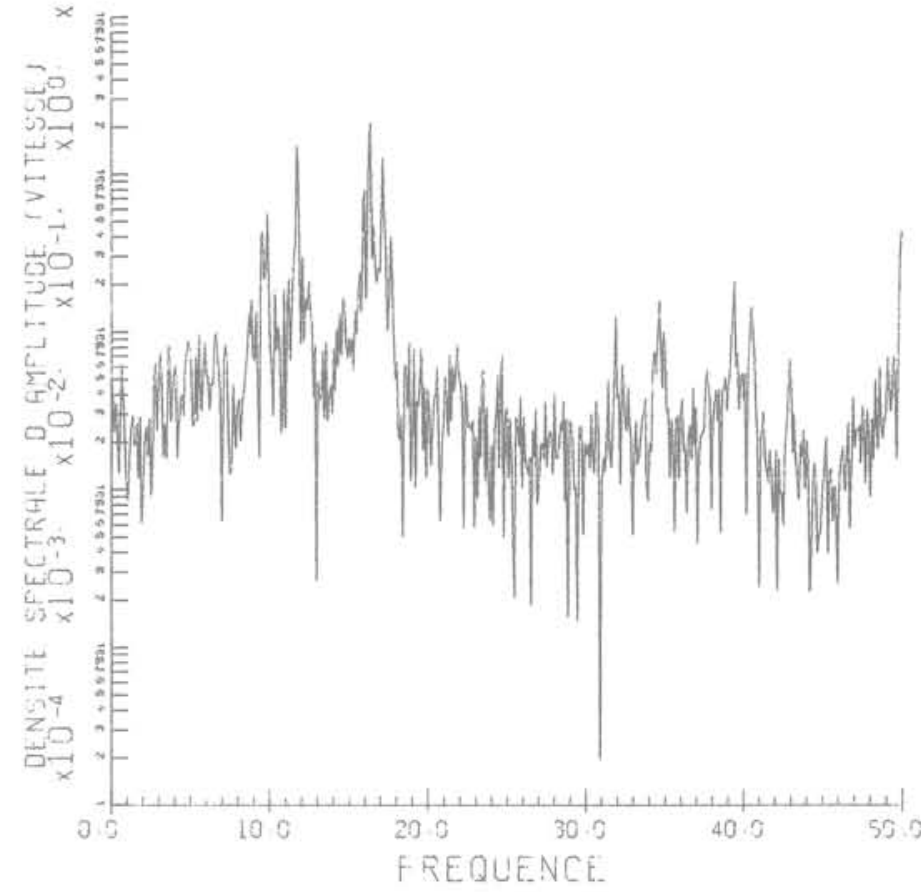

Figure 3. - Exemple de spectre d'amplitude pour la composante verticale des mouvements stationnaires de la station 4.

Un maximum très marqué est également observé à $40 \mathrm{~Hz}$; ce dernier n'a pas été observé sur la station 1 et nous ne l'avons pas retenu comme caractéristique. Nous avons essayé d'effectuer une analyse similaire pour la station 3 , mais le niveau moyen de bruit, beaucoup plus élevé que pour la station 4 du fait dela présence d'engins de terrassement travaillant dans la carrière à moins de $200 \mathrm{~m}$ de la station, ne fournit pas une conclusion aussi nette (voir figure 4).

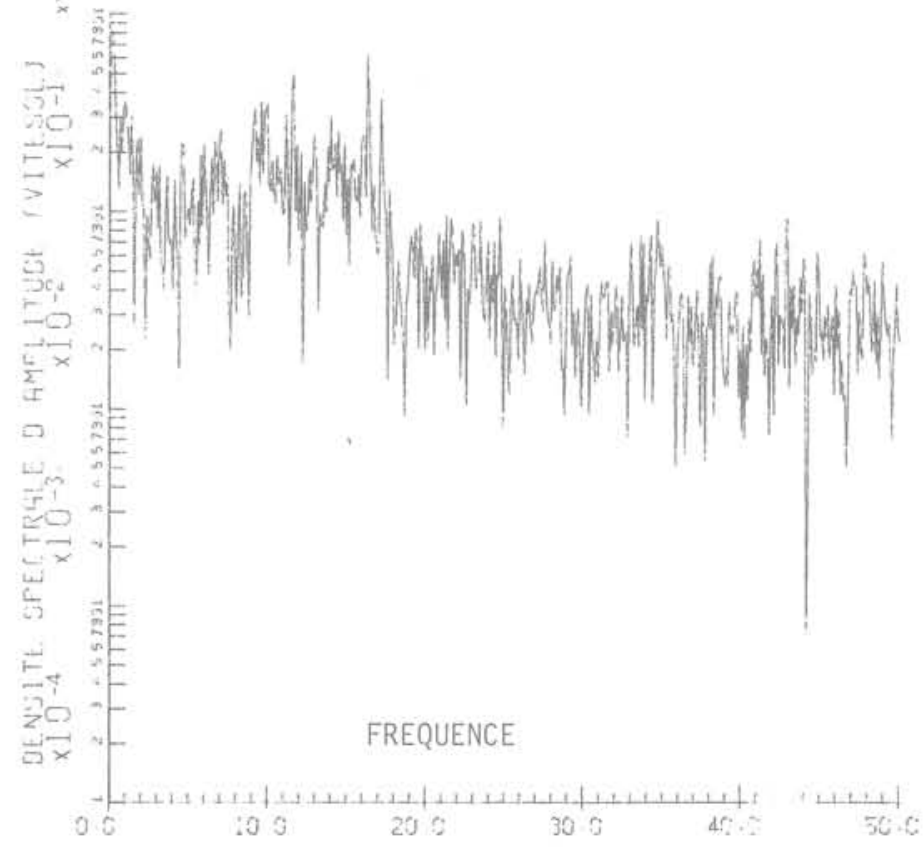

Fizure 4. - Exemple de spectre d'amplitude pour la composante verticale des mouvements stationnaires de la station 3. 
I1 peut être intéressant de noter que sur la station 4 les vibrations liêes aux concasseurs sont nettement plus visibles, ceci provient probablement de ce que le terrain sous-jacent en 4 est un poudingue mal consolidé alors qu'il est constitué de calcaire homogène en 3 .

\section{Attênuation des mouvements vibratoires générês păr la carrière:}

La station 3 est un bon exemple de la difficulté que 1 'on rencontre à définir précisément le domaine dans lequel les vibrations générées par les unités de concassage sont sensibles: les fréquences caractéristiques ne peuvent être individualisées qu'au moyen de leur amplitude relative par rapport au niveau de bruit moyen ambiant. Dans un environnement très bruyant, sur un large domaine de fréquence, il devient impossible d'individualiser la contribution de la carrière, la définition de la limite du domaine affecté par les vibrations des concasseurs dépend donc du niveau moyen de bruit.

Nous avons mesuré systématiquement les niveaux moyens de bruit pour les domaines $1-10 \mathrm{~Hz}, 10-20$ $\mathrm{Hz}, 30-40 \mathrm{~Hz}$ et $40-50 \mathrm{~Hz}$, simultanément avec les amplitudes maximum observées dans les domaines 8-10 Hz, $12 \mathrm{~Hz}, 16-18 \mathrm{~Hz}, 20-22 \mathrm{~Hz}$ pour chacune des stations. La détermination du niveau moyen de bruit a été conduite de façon empirique : on a essayé visuellement de définir un niveau d'amplitude moyen tel que la somme des différences d'amplitudes par rapport à ce niveau soit le même pour les amplitudes supérieures et pour les amplitudes inférieures. Une méthode numérique rigoureuse aurait pu être mise au point; toutefois l'amélioration sur la précision de la valeur ainsi obtenue n'aurait pas modifié nos conclusions.

Pour les stations 6 et 8 situées environ à $1500 \mathrm{~m}$ des concasseurs on retrouve très nettement les fréquences $8-10 \mathrm{~Hz}$ et $16-18 \mathrm{~Hz}$. Les niveaux d'amplitude sont néanmoins très différents d'une station à l'autre, ce qui souligne bien le rôle d'une part de la nature des terrains séparant le site considêré de la source, d'autre part de la nature du terrain au niveau du site même.

Ces piques d'amplitude sont encore visibles ã $3 \mathrm{~km}$ sur toutes les stations $(9,10,11$ et 12) ; cependant, pour les stations 9 et 10 , seules les composantes horizontales sont encore affectées (la station 9 repose directement sur le calcaire alors que la station 10 est séparée du calcaire par une couche de terre d'épaisseur inconnue). Pour la station 12, une autre source se superpose qui gêne la lecture du maximum pour la fréquence $9 \mathrm{~Hz}$, celle-ci reste néanmoins visible.

Sur aucune des stations à $7 \mathrm{~km}$ l'activitê de la carrière n'est visible. Certes, un pique très marquê pour la station 16 a pu être observé à la fréquence $16 \mathrm{~Hz}$ pour la première, et surtout 1a deuxième fenêtre d'échantillonage mais $i 1$ n'est pas apparu pour les autres fenêtres. De plus, le niveau très êlevé de ce pique, supérieur à celui observé à $3 \mathrm{~km}$ dans la même direction, permet de conclure qu'il est associé à une autre activité humaine. On relève en effet au voisinage de cette station de nombreux chantiers de construction. Un pique à $20 \mathrm{~Hz}$ observé sur la station 15 est à attribuer aux travaux effectuês, à moins d'un kilomètre du site, au moyen d'une machine rotative destinée à creuser une tranchée étroite. Ces résultats sont résumés sur le tableau 1 .

\section{ETUDE DU MOUVEMENT ASSOCIE A UN TIR DE MINE}

Deux questions étaient posées en ce qui'concerne les tirs de mine qui ont lieu trois à quatre fois par semaine.

1. Le mouvement peut-il être ressenti sur un site situé à $7 \mathrm{~km}$ ?

2. Le mouvement peut-il générer des déformations permanentes?

Un enregistrement a été effectué sur les stations $6,10,14$ et 17 lors d'un tir normal (535 kg de dynamite gomme en 6 charges avec des micro-retards entre chaque charge de 0,005 seconde) effectué dans la carrière (voir figure 5.); elles étaient disposées sur une même ligne droite issue de la carrière et à des distances respectivement de $1000 \mathrm{~m}, 2500 \mathrm{~m}$, $7750 \mathrm{~m}, 15800 \mathrm{~m}$ du point de tir.

L'enregistrement à la station $14(7750 \mathrm{~m}$ du point de tir) est très net ; la durée du mouvement y est d'environ 5 secondes. L'essentiel de l'énergie se trouve dans le domaine de fréquence $1-20 \mathrm{~Hz}$ et les amplitudes maximales des composantes verticales, longitudinales, et transversales de la vitesse sont respectivement de $7,9 \mu \mathrm{m} / \mathrm{s}, 18,9 \mu \mathrm{m} / \mathrm{s}$ et $14,2 \mu \mathrm{m} / \mathrm{s}$. Ces mouvements correspondent à des déformations inférieures à $10^{-7}$ (déplacements maximum de 1'ordre du micron pour des longueurs d'onde de 1 'ordre de la centaine de mètres), ce qui reste largement dans le domaine élastique du comportement des terrains de fondation.

\section{CONCLUSIONS}

Cette étude avait pour but de préciser la façon dont sont ressentis les mouvements vibratoires générés dans le sol par l'exploitation d'une carrière.

Une station d'enregistrement implantẻe à $100 \mathrm{~m}$ des unitês de concassage a permis de préciser le domaine de fréquence des vibrations générées par celles-ci Les domaines $8-10 \mathrm{~Hz}, 12 \mathrm{~Hz}, 16-18 \mathrm{~Hz}$ et $20-22 \mathrm{~Hz}$ sont affectês de façon très significative. Les vibrations sont encore ressenties bien que de façon très ténue, à $3 \mathrm{~km} 500$ de la carrière, mais ne sont plus discernables à $7 \mathrm{~km}$.

L'enregistrement à $1000 \mathrm{~m}, 2500 \mathrm{~m}, 7750 \mathrm{~m}$ et $15800 \mathrm{~m}$ des vibrations générées par un tir de $535 \mathrm{~kg}$ de dynamite gomme en 6 charges avec des micro-retards entre chaque charge de 0,005 seconde permet d'illustrer la façon dont s'atténue le signal avec la distance pour des terrains calcaires relativement homogênes. A ces distances, le terrain présente une réponse élastique pour ces sollicitations dynamiques. 


\section{REMERC IEMENTS}

Cette étude a étē financẻe par l'entreprise Spada de îice. Le matêriel d'enregistrement êtait celui de l'équipe des grands profils sismiques de I'IPGP.

\begin{tabular}{|c|c|c|c|c|c|c|}
\hline \multirow{2}{*}{$\begin{array}{c}\text { Station } \\
n^{\circ}\end{array}$} & \multirow{2}{*}{$\begin{array}{l}\text { Distance } \\
\text { en } \mathrm{m} \text {. }\end{array}$} & \multicolumn{5}{|c|}{ Amplitude moyenne en $\mu \mathrm{m} / \mathrm{s}$, pour les frếquences. } \\
\hline & & $8-10 \mathrm{~Hz}$ & & $12 \mathrm{~Hz}$ & $15-18 \mathrm{~Hz}$ & $20-22 \mathrm{~Hz}$ \\
\hline $\begin{array}{r}1 \\
4 \\
3 \\
6 \\
8 \\
10 \\
12 \\
11\end{array}$ & $\begin{array}{r}100 \\
772 \\
934 \\
1484 \\
1494 \\
2855 \\
3021 \\
3422\end{array}$ & $\begin{array}{l}1,1 \pm 0,4 \\
0,5 \pm 0,05 \\
0,4 \pm 0,1 \\
0,09 \pm 0,04 \\
0,07 \pm 0,01 \\
=- \\
0,03 \pm 0,003 \\
0,04 \pm 0,02\end{array}$ & $\begin{array}{l}0,4 \\
1,7 \\
0,4\end{array}$ & $\begin{array}{l} \pm 0,05 \\
\pm 0,2 \\
+0,1 \\
- \\
- \\
- \\
- \\
-\end{array}$ & $\begin{array}{l}0,8 \pm 0,2 \\
2,1 \pm 0,2 \\
0,7 \pm 0,1 \\
0,04 \pm 0,01 \\
0,3 \pm 0,1 \\
= \\
0,1 \pm 0,06 \\
0,06 \pm 0,03\end{array}$ & $\begin{aligned} & 0,8 \pm 0,3 \\
& 0,2 \pm 0,1 \\
& 0,4 \pm 0,05 \\
&= \\
&- \\
&= \\
& 0,06 \pm 0,04\end{aligned}$ \\
\hline
\end{tabular}

Tableau 1 : Variation, en fonction de la distance site-source, de I'amplitude de la composante verticale de la vitesse du mouvement stationnaire généré par une unité de concassage et observéesur des stations distribuées dans un secteur angulaire de $90^{\circ}$.

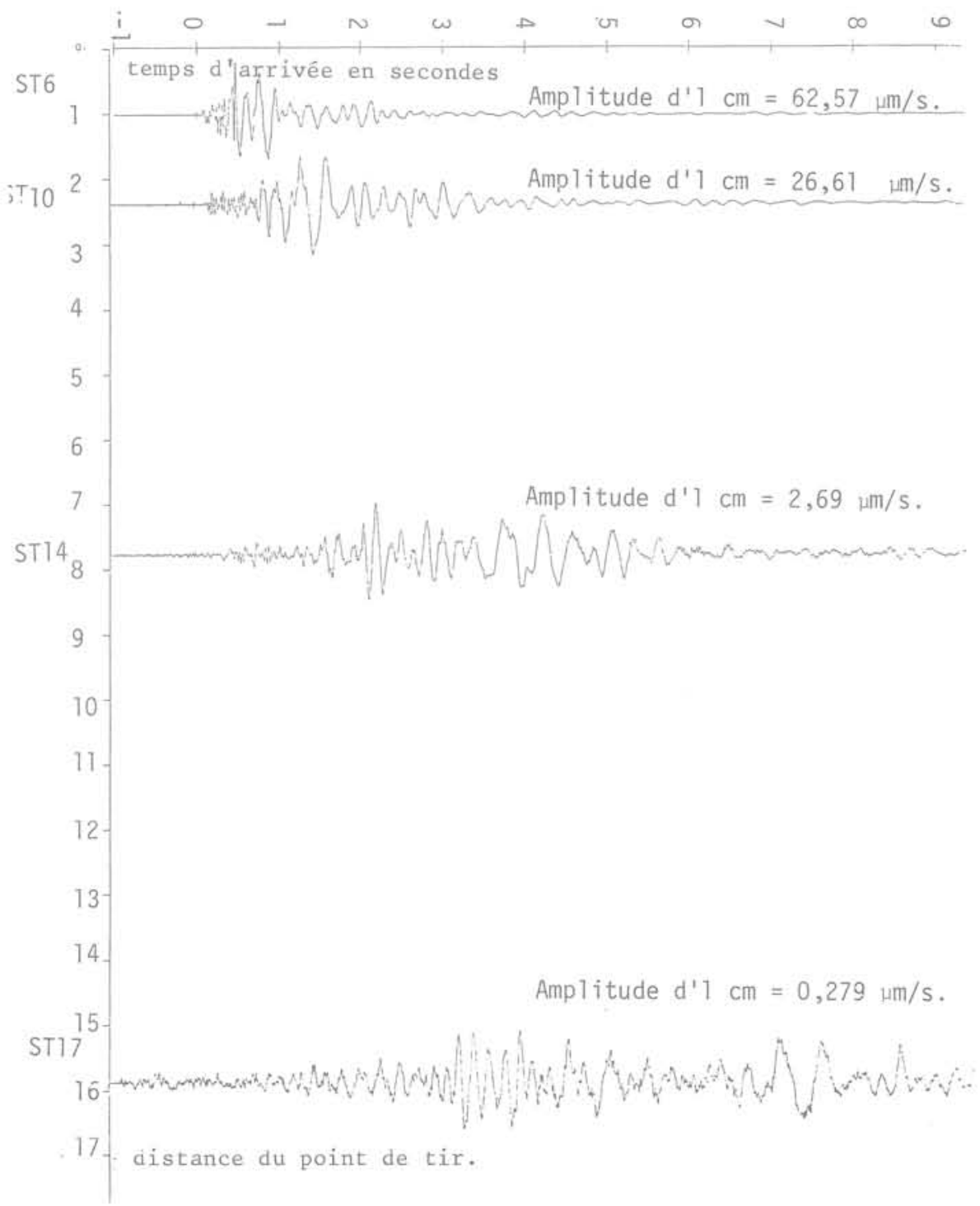

Figure 5 : Composante verticale du mouvement gênéré par un tir de $535 \mathrm{Kg}$ de dynamite gomme avec 6 micro-retards de 0,005 seconde et ressentis aux stations $6,10,14$ et 17 . Le temps $t=0$ est choisi à l'instant de la détonation. 